\title{
Emulsified silicone oil is taken up by and induces pro-inflammatory response in primary retinal microglia
}

\author{
Alexa Klettner $^{1}$ (D) Antonia Harms ${ }^{1} \cdot$ Vicki Waetzig $^{2} \cdot$ Jan Tode $^{1} \cdot$ Konstantine Purtskhvanidze $^{1} \cdot$ Johann Roider $^{1}$
}

Received: 14 August 2019 / Revised: 7 April 2020 / Accepted: 20 May 2020 / Published online: 4 June 2020

(C) The Author(s) 2020

\begin{abstract}
Purpose Silicone oil is used as endotamponade in combination with vitrectomy. Thinning of retinal layers and loss of retinal cells under silicone oil use have been found. Here, we investigate the influence of silicone oil on primary microglia cells.

Methods Primary microglia cells were prepared from the porcine retina. Microglia identity was assessed with Iba1 staining. Silicone oil was emulsified by sonification. Cell morphology and silicone oil uptake were evaluated by light microscopy after Coomassie blue staining. Cytokine secretion was evaluated with ELISA. Toxicity of silicone oil on microglia and toxic effect of silicone oil-treated microglia on neuronal cell line PC12 were evaluated by MTT or WST assay, respectively.

Results Microglia took up silicone oil droplets after $72 \mathrm{~h}$ of incubation. Silicone oil induced no toxicity but increased the metabolism in microglial cells. In addition, the secretion of IL- 6 and IL- 8 , but not of IL-1ß or TNF- $\alpha$, was induced. Silicone oil-treated microglia did not exert any neurotoxic effect on differentiated PC12 cells but induced an increase in metabolism.

Conclusion Emulsified silicone oil changes the activity level of microglia and induces the secretion of IL-6 and IL-8. Neurotoxicity is not induced. Further experiments are required to investigate the long-term effect of silicone oil on microglia and their consequent effect on neuronal cells.
\end{abstract}

Keywords Silicone oil · Endotamponade · Microglia · IL-6 · IL-8

\section{Introduction}

In the management of severe vitreoretinal diseases such as retinal detachment with proliferative vitroretinopathy, giant retinal tear, or multiple retinal tears, silicone oil is widely used as long-term endotamponade.

Due to stability, immunological tolerability, and lack of direct toxicity, it is generally regarded as a safe compound and can therefore be used as a long-acting tamponade [1, 2]. Silicone oil has been in clinical use for more than 30 years, mostly in complicated cases or in reoperations. A variety of complications have been described for the use of silicone oil, including cysts, emulsification, and migration of the oil [3].

Alexa Klettner

AlexaKarina.Klettner@uksh.de

1 Department of Ophthalmology, University Medical Center, University of Kiel, Arnold-Heller-Str. 3, Haus 25,

24105 Kiel, Germany

2 Department of Pharmacology, University Medical Center, University of Kiel, Hospitalstr. 4, 24105 Kiel, Germany
Therefore, it is recommended to remove silicone oil within a period of 3 months up to 1 year. Recently, severe loss of visual acuity has been - initially anecdotally — reported in individual cases. Detailed studies and studies on a very well-documented patient population indicate that a severe loss in visual acuity might occur much more frequently than generally assumed. They showed that in $20-30 \%$ of patients treated with silicone oil visual acuity is much worse than could be expected $[4,5]$. In such cases, all the clinical investigations, e.g., fluorescein angiography, OCT, or analysis of the operating report, did not give a satisfactory explanation for the decline in visual acuity. Tode et al. have shown that this unexplained loss was accompanied by a thinning of foveal and parafoveal combined nerve fiber, ganglion cell, and inner plexiform layers.

The mechanisms behind this pathology are not known so far. In earlier studies, silicone oil-associated vision loss was discussed to be associated with silicone oil droplets found in the retina and optic nerve [6]. Silicone oil tends to emulsify in retinal tissue as saccadic, and pursuit eye and head movements cause shear stress on silicone oil bubbles [7]. While these bubbles are generally considered to be transient, they may become permanent in the presence of blood components or 
pro-inflammatory proteins [2]. Contrary to the immunological tolerability attributed to silicone oil, emulsified silicone oil bubbles are suspected to trigger inflammation, phagocytosis, and chemotaxis [2]. These cases usually have a history of complicated surgery or pathology. However, the causes of vision loss in cases with or without such a history are not known so far. Roider et al. postulated some immunological reactions in the fovea due to the exposure of microglial cells to silicone oil (VAIL-Vitrectomy 2016).

Silicone oil has previously been described to be incorporated by macrophages, which was linked to transport of silicone oil into the retina, optic nerve, or brain [8-10]. Bloodborne inflammatory-activated macrophages are found in epiretinal membranes of silicone oil-treated eyes but hardly in the corresponding retina [11]. This correlates well to the fact that the innate immune cell of the retina is the microglia, not the macrophage. Microglia are not blood-borne but migrate into the retina from the yolk sac during embryogenesis [12]. In healthy retinas, microglia are located in the inner retinal layers, constantly surveying their surroundings, while upon activation, they become motile, pro-inflammatory, and phagocytic. Involvement of microglia in retinal degenerative diseases is widely discussed especially as prolonged activated microglia may become neurotoxic [13]. Microglial interaction with silicone oil has not been investigated so far. In this study, we have treated primary retinal microglia with emulsified silicone oil, investigating toxicity, morphology, and proinflammatory cytokine release.

\section{Materials and methods}

\section{Microglia cells}

Primary microglia were prepared from the porcine retina as described [14]. For experimentation, cells were harvested and seeded at a cell density of 250,000 cells/well in 24-well (Sarstedt, Nümbrecht, Germany) or 96-well plates (Roth, Karlsruhe, Germany). Cell number was assessed with trypan blue exclusion assay. Twenty-four hours after seeding, the medium was changed and the appropriate substance was added. Emulsified silicone oil (1\%, 5\%, 10\%; Oxane 5700, Bausch \& Lomb GmbH, Berlin, Germany) was incubated for 24-72 h. Polyinosinic:polycytidylic acid (Poly I:C; Tocris, Bristol, UK; $100 \mu \mathrm{g} / \mathrm{ml}$ ) was used as a positive control for microglia stimulation [15].

\section{PC12 cells}

Differentiated rat pheochromocytoma PC12 cells are a widely used cell culture model for neuronal cells [16], especially concerning neurotoxicity and neuroprotection [17]. These cells were directly obtained from the German Collection of
Microorganisms and Cell Cultures (DSMZ), Braunschweig, Germany. No subclones were used. Cells were cultured on collagen-coated plates in RPMI 1640 medium (Life Technologies; Darmstadt, Germany) supplemented with 5\% fetal bovine serum (FBS; Biochrom; Berlin, Germany) and $10 \%$ horse serum (Biochrom) at $37{ }^{\circ} \mathrm{C}$ and $5 \% \mathrm{CO}_{2}$. For neuronal differentiation, $\mathrm{PC} 12$ cells were plated at low density and kept in serum-containing medium for $24 \mathrm{~h}$, cultured in medium supplemented with $0.5 \%$ FBS and $1 \%$ penicillin/ streptomycin for additional $72 \mathrm{~h}$, and treated with nerve growth factor (NGF) $(50 \mathrm{ng} / \mathrm{ml}$; Alomone Labs; Jerusalem, Israel) for seven days. Fresh NGF was added every second day [18].

\section{Fluorescence microscopy}

Iba1 is considered a marker for microglial cells [13]; therefore, Iba1 staining was conducted to assess the purity of the cells as previously described [14]. Per slide, eight pictures were taken and cell nuclei and Iba1 fluorescent cells were assessed. The number of nuclei was set in relation to Iba1 positive-stained cells.

\section{Emulsification of silicone oil}

Silicone oil was added in a concentration of $1 \%, 5 \%$, or $10 \%$, respectively, in cell culture medium (DMEM, supplemented with $10 \% \mathrm{FBS}$ and $1 \%$ penicillin/streptomycin) and vortexed. The mixture was sonicated for $30 \mathrm{~min}$ in a sonication bath (Ultrasonic Cleaner USC-TH VWR, Darmstadt). Bubble formation was verified in light microscopy.

\section{Coomassie staining}

For light microscopic evaluation of morphology and silicone oil uptake, cells were stained with Coomassie. After incubation and removal of the supernatant, cells were washed with PBS and fixed for 40 min with $2.5 \%$ glutaraldehyde (Merck, Darmstadt, Germany). Cells were washed with PBS and stained for 10 min with Coomassie Brilliant Blue R-250 staining solution (Bio-Rad, München, Germany) and destained for $2 \times 20$ min with Coomassie Brilliant Blue R-250 destaining solution (Bio-Rad). Afterwards, cells were washed three times with PBS and once with Aqua Dest (Ampuwa, Fresenius, Bad Homburg, Germany) and mounted with Faramount (Dako, Hamburg, Germany).

\section{Microglia morphology}

Microglia were categorized according to morphological features [19]. "Resting" microglia presented as small cells (100$500 \mu^{2}$ ) with many ramifications. "Ameboid" cells presented as larger $\left(500-1000 \mu \mathrm{m}^{2}\right)$, plump cells that had lost their 
ramification. A third category is the large "giant" cells [20], with a size above $1000 \mu \mathrm{m}^{2}$ and polymorphic cell shape.

\section{Silicone oil inclusions}

Coomassie-stained microglia were analyzed in light microscopy. For all cover slips, nine pictures were taken in a fixed pattern and the silicone oil droplets per cell were assessed. A silicone oil droplet had to present as a clearly bordered intracellular inclusion that was devoid of blue staining $(\times 20$ magnification). Inclusions were not regarded as silicone oil droplets if they did not present with a clear border to the adjunct cell plasma.

\section{MTT assay}

In order to test the direct toxicity of silicone oil on the microglia cells, an MTT assay (3-(4,5-dimethylthiazol-2yl)2,5diphenyltetrazoliumbromid) was conducted [21]. Cells were incubated with different concentrations of emulsified silicone oil for 24, 48, or $72 \mathrm{~h}$ and incubated with MTT (Sigma-Aldrich, Steinheim, Germany) in DMEM (without phenol red) for $2 \mathrm{~h}$. Supernatant was discarded, and DMSO (Roth) was added to the cells and rocked. The extinction of the supernatant was measured at a wavelength of $550 \mathrm{~nm}$ (ELx800, BioTek, Bad Friedrichshall, Germany), and DMSO served as a blank control. Untreated cells served as control and were set at $100 \%$.

\section{WST assay}

To test the toxic effects of the supernatant of microglial cells on PC12 cells, a WST (water-soluble tetrazolium) assay was conducted. In this test, the activity of the superoxide dismutase is detected, as a surrogate parameter for cell activity [22]. Cells were grown on 96 -well plates $\left(2 \times 10^{6}\right.$ cells per well $)$ and treated for $48 \mathrm{~h}$ with the supernatant of microglia that had been treated with emulsified silicone oil for $72 \mathrm{~h}$. As respective controls, $\mathrm{PC} 12$ cells were treated with supernatant of untreated microglia, emulsified silicone oil without microglia medium, or medium without further treatment. After the experiment, the medium was discarded and $10 \mu \mathrm{l}$ of watersoluble tetrazolium-1 (WST-1) reagent (Sigma; Munich, Germany) was added to each well to determine metabolic activity. The cells were incubated for $2 \mathrm{~h}$ at $37{ }^{\circ} \mathrm{C}$ and $5 \%$ $\mathrm{CO}^{2}$. The plate was placed into a Tecan infinite M200 reader (Tecan; Crailsheim, Germany) and shaken thoroughly for 1 min. Absorbance was measured at $450 \mathrm{~nm}$ with a reference wavelength of $600 \mathrm{~nm}$. Per plate, one well with cells treated only with medium without further treatment was set as $100 \%$, and the other values were calculated accordingly.
Enzyme-linked immunosorbent assay (ELISA)

ELISAs were conducted to detect IL-6, IL-1ß, IL-8, and TNF- $\alpha$ content in the supernatant. All kits were obtained from R\&D Systems (porcine IL-6: \#P6000B; porcine CXCL8/IL-8: \#P8000, porcine IL-1B/IL-1F2: \#PLB00B; porcine TNF- $\alpha$ : \# PTA00) and conducted according to the manufacturer's instructions.

\section{Statistics}

Each experiment was independently repeated at least 3 times. Bar graphs depict mean and standard deviation. Significance was evaluated with Friedman's ANOVA for three and more groups with post-hoc Wilcoxon's test. Student's $t$ test was conducted for the comparison of two groups. Statistical analysis has been conducted using Statistica (7.1 StatSoft, Inc.) and Microsoft Excel.

\section{Results}

\section{Identity of microglia cells: Iba1 staining}

The expression of Iba1 was assessed on microglia seeded on cover slips with a mean of 91.5\% positive cells (Fig. 1, 200 cells assessed). Iba1 negative nuclei presented as condensed nuclei with brilliant blue staining indicating cell death [23], rather than contaminating cells.

\section{Microglia morphology}

In untreated control cells after $24 \mathrm{~h}$ in culture, $76.52 \%( \pm$ $11.31)$ of the microglia present as resting, $14.10 \%( \pm 7.69)$ of the microglia present as amoeboid and 9.49\% $( \pm 5.30)$ of the cells present as giant (Fig. 2a, c; 3591 cells assessed). When treated with Poly I:C, the percentage of resting microglia significantly decreased $(p<0.01)$ while the number of amoeboid cells increased $(p<0.01)$ (Fig. 2b; 3087 cells assessed). After treatment with 5\% or $10 \%$ silicone oil, respectively, no significant changes between the groups ( $24 \mathrm{~h}$ (Fig. 2d; 4980 cells assessed), $48 \mathrm{~h}$ (Fig. 2e; 9256 cells assessed) or $72 \mathrm{~h}$ (Fig. 2f; 20212 cells assessed)) could be detected in Friedman's ANOVA (Table 1).

\section{Toxicity of silicone oil on microglia cells}

Toxicity of emulsified silicone oil in microglia cells was tested with an MTT viability assay. No decrease in viability could be found for $5 \%$ or $10 \%$ silicone oil after 24,48 , or $72 \mathrm{~h}$ (Fig. 3a). Of note, a significant increase of MTT signal as detected by Friedman's ANOVA and post-hoc Wilcoxon's test after $72 \mathrm{~h}$ $(p<0.05)$, suggesting an activation of the cells (Table 2$)$. We 
Fig. 1 Iba1 staining for the identification of microglia. In immunofluorescence staining, a mean of $91.5 \%$ of the cells stained positive for microglia marker Iba1 ( $n=200$ cells, 8 independent experiments). Note the chromatin dense structure of negative nuclei (upper arrow of magnification), indicating that these structures are apoptotic cells, not contaminating cell types

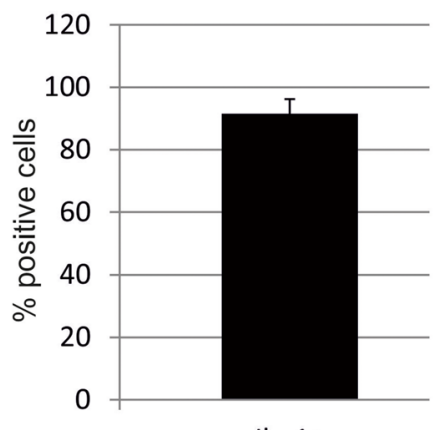

Iba1+

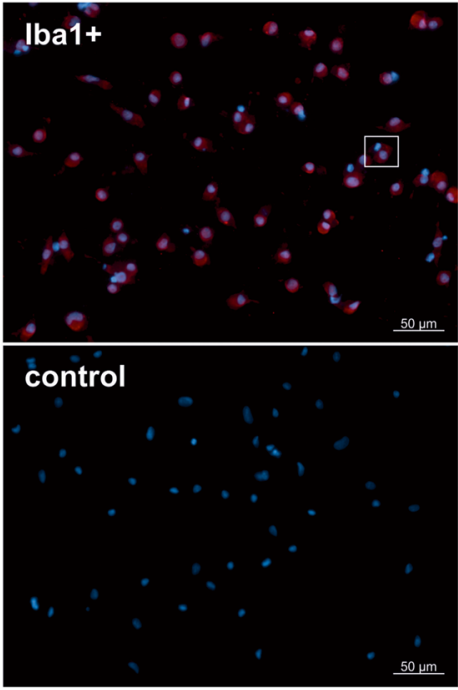

negative nucleus

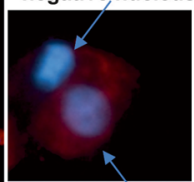

positive cell a) Morphological distribution untreated microglia (24 h)

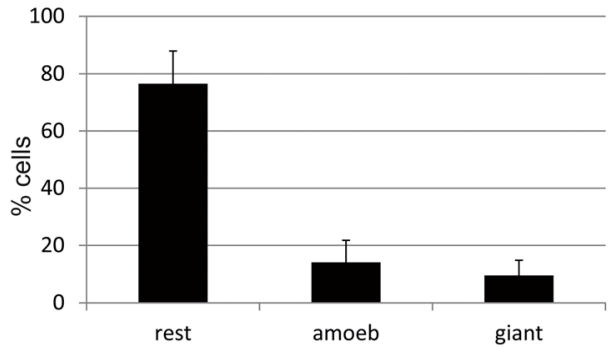

b) Morphological distribution of microglia with Poly I:C (24 h)

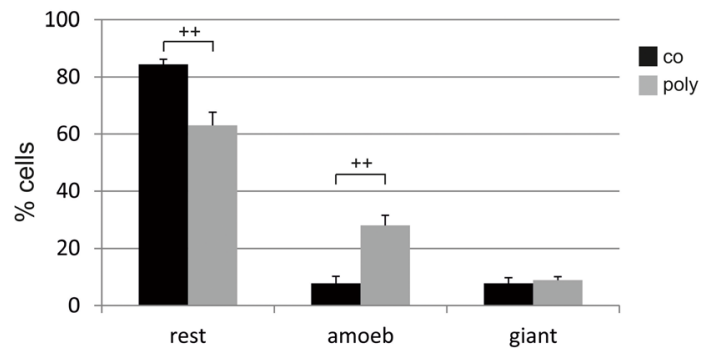

c) Exemplary pictures of resting, amoeboid and giant cells

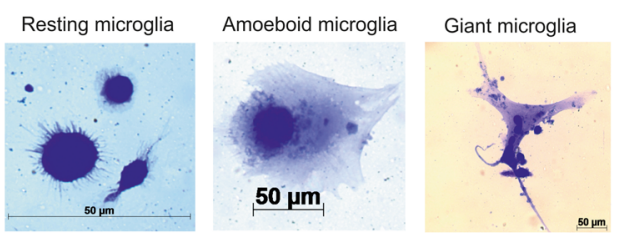

d) Morphological distribution of microglia with silicone oil (24 h)

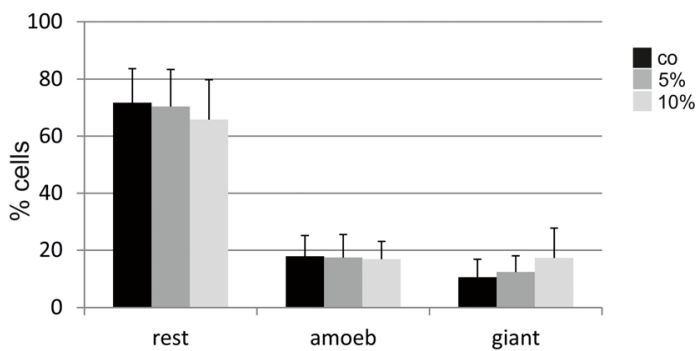

e) Morphological distribution of microglia with silicone oil (48 h)

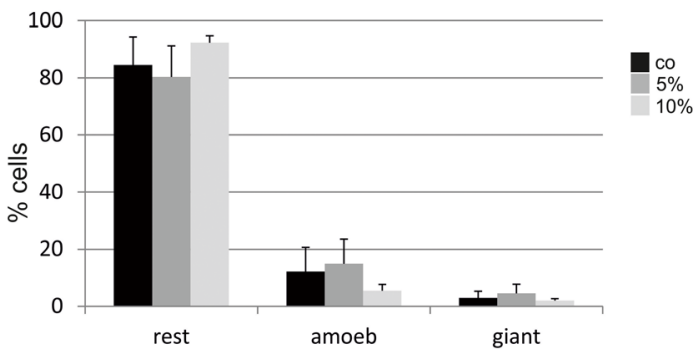

f) Morphological distribution of microglia with silicone oil (72 h)

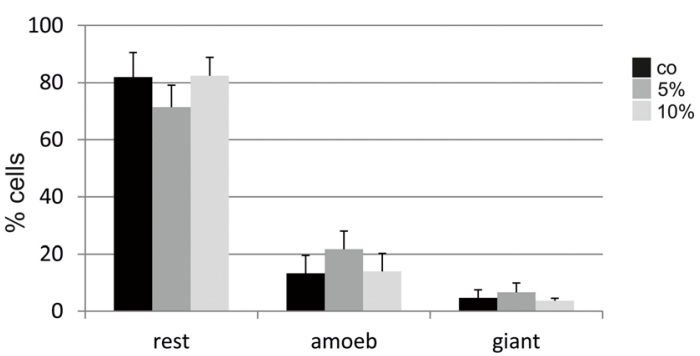

After $24 \mathrm{~h}$ (d), $48 \mathrm{~h}$ (e), and $72 \mathrm{~h}$ (f), no changes can be observed. Significance was evaluated with Friedman's ANOVA for a comparison of 3 groups or Student's $t$ test, double positive sign indicates $p<0.01$. Co, control; rest, resting microglia; amoeb, amoeboid microglia; giant, giant microglia 
Table 1 Morphology of microglia after treatment with 5\% or $10 \%$ emulsified silicone oil

\begin{tabular}{|c|c|c|c|c|c|c|c|c|}
\hline Time (h) & Morphology & Treatment & Rank & Rank sum & Median & Minimum & Maximum & Friedman \\
\hline \multirow[t]{9}{*}{24} & \multirow[t]{3}{*}{ Resting } & Control & 2.25 & 9 & 19.53 & 4.48 & 24.63 & \multirow[t]{3}{*}{ n.s. } \\
\hline & & $5 \%$ & 1.75 & 7 & 16.21 & 14.24 & 23.1 & \\
\hline & & $10 \%$ & 2 & 8 & 14.42 & 11.62 & 27 & \\
\hline & \multirow[t]{3}{*}{ Amoeboid } & Control & 2.25 & 9 & 19.53 & 4.48 & 24.63 & \multirow[t]{3}{*}{ n.s. } \\
\hline & & $5 \%$ & 1.75 & 7 & 16.21 & 14.24 & 23.71 & \\
\hline & & $10 \%$ & 2 & 8 & 14.42 & 11.62 & 27 & \\
\hline & \multirow[t]{3}{*}{ Giant } & Control & 2 & 8 & 8.3 & 3.05 & 20.31 & \multirow[t]{3}{*}{ n.s. } \\
\hline & & $5 \%$ & 2 & 8 & 7.88 & 5.75 & 28.48 & \\
\hline & & $10 \%$ & 2 & 8 & 14.555 & 6.32 & 33.96 & \\
\hline \multirow[t]{9}{*}{48} & \multirow[t]{3}{*}{ Resting } & Control & 1.666667 & 5 & 83.25 & 67.97 & 94.63 & \multirow[t]{3}{*}{ n.s. } \\
\hline & & $5 \%$ & 1.333333 & 4 & 84.77 & 65.41 & 92.79 & \\
\hline & & $10 \%$ & 3 & 9 & 91.5 & 89.9 & 95.67 & \\
\hline & \multirow[t]{3}{*}{ Amoeboid } & Control & 2.666667 & 8 & 9.1 & 4.29 & 27.12 & \multirow[t]{3}{*}{ n.s. } \\
\hline & & $5 \%$ & 2.333333 & 7 & 12.5 & 3.9 & 25.71 & \\
\hline & & $10 \%$ & 1 & 3 & 5.59 & 2.89 & 8.21 & \\
\hline & \multirow[t]{3}{*}{ Giant } & Control & 2 & 6 & 1.32 & 0.87 & 6.63 & \multirow[t]{3}{*}{ n.s. } \\
\hline & & $5 \%$ & 2.666667 & 8 & 3.3 & 1.84 & 10.78 & \\
\hline & & $10 \%$ & 1.333333 & 4 & 1.89 & 1.44 & 2.96 & \\
\hline \multirow[t]{9}{*}{72} & \multirow[t]{3}{*}{ Resting } & Control & 2.2 & 11 & 75.8 & 70.8 & 94.118 & \multirow[t]{3}{*}{ n.s. } \\
\hline & & $5 \%$ & 1.4 & 7 & 73.68 & 58.34 & 82.62 & \\
\hline & & $10 \%$ & 2.4 & 12 & 86.075 & 73.32 & 90.408 & \\
\hline & \multirow[t]{3}{*}{ Amoeboid } & Control & 1.6 & 8 & 17.59 & 5.11 & 21.91 & \multirow[t]{3}{*}{ n.s. } \\
\hline & & $5 \%$ & 2.6 & 13 & 20.89 & 14.06 & 29.74 & \\
\hline & & $10 \%$ & 1.8 & 9 & 9.968 & 6.38 & 23.89 & \\
\hline & \multirow[t]{3}{*}{ Giant } & Control & 1.833333 & 11 & 4.8325 & 6.049 & 0.47 & \multirow[t]{3}{*}{ n.s. } \\
\hline & & $5 \%$ & 2.666667 & 16 & 6.651167 & 6.805 & 2.33 & \\
\hline & & $10 \%$ & 1.5 & 9 & 3.6626 & 3.8678 & 2.235 & \\
\hline
\end{tabular}

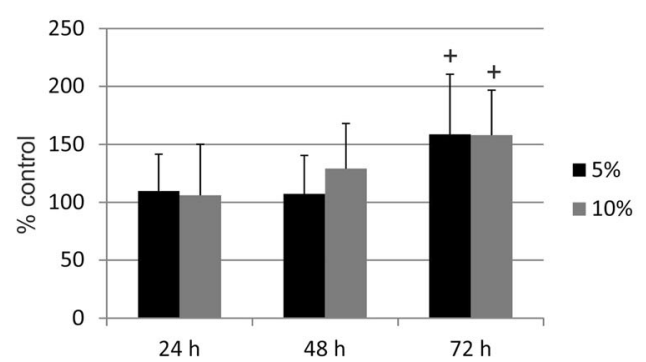

Fig. 3 Cell viability testing. a Toxicity of emulsified silicone oil on microglia cells was tested after 24,48 , and $72 \mathrm{~h}$ for $5 \%$ and $10 \%$ silicone oil with MTT assay. No toxicity could be detected. Untreated control at the respective time point was set as $100 \%$ and is not depicted in the graph. Of note, after $72 \mathrm{~h}$, a significant induction of the signal could be found for 5\% $(p<0.05)$ and $10 \%(p<0.01)$ compared with untreated control. b Cell viability of silicone oil-treated microglia on PC12 cells was tested with WST assay. Microglia were treated for $72 \mathrm{~h}$ with emulsified silicone oil, the supernatant was collected, and PC12 cells were incubated with the microglia supernatant for $48 \mathrm{~h}$. In addition, PC12 cells were either treated with microglia medium without silicone oil treatment or directly with emulsified silicone oil. Untreated cells (no microglia, no

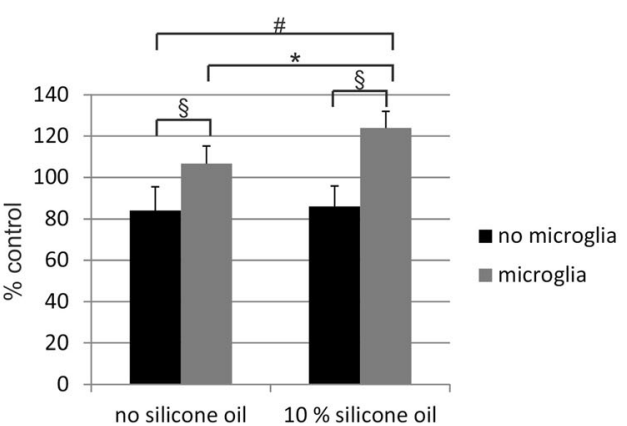

silicone oil) served as controls. No decline in cell viability could be detected. However, a significant elevation of the PC12 signal could be found when cells were treated with supernatant of microglia exposed to silicone oil, indicating increased metabolic activity. Significance was evaluated with Friedman's ANOVA and Wilcoxon's test. a Positive sign indicates $p<0.05$ against untreated control. $\mathbf{b}$ The section sign indicates $p$ $<0.05$ comparing cells with and without microglia supernatant treatment. Asterisk indicates $p<0.05$ comparing cells treated with microglia supernatant with or without silicone oil, and number sign indicates $p<0.05$ comparing untreated cells with cells treated with supernatant of microglia treated with silicone oil 
Table 2 Viability of microglia after treatment with $5 \%$ or $10 \%$ emulsified silicone oil

\begin{tabular}{llllllll}
\hline Time (h) & Treatment & Rank & Rank sum & Median & Minimum & Maximum & Friedman \\
\hline 24 & Control & 1.8 & 9 & 100 & 100 & 100 & Wilcoxon \\
& $5 \%$ & 2.4 & 12 & 126.9 & 71.4 & 148.2 & \\
& $10 \%$ & 1.8 & 9 & 117.5 & 49.9 & 171.6 & n.s. \\
48 & Control & 1.14286 & 12 & 100 & 100 & 100 & \\
& $5 \%$ & 1.714286 & 12 & 102.38 & 51.28 & 163.13 & \\
72 & $10 \%$ & 2.571429 & 18 & 123.2 & 73.08 & 181.8 & $p<0.05$ \\
& Control & 1.142857 & 8 & 100 & 100 & 100 & $p<0.05$ \\
& $5 \%$ & 2.428571 & 17 & 125.64 & 106.25 & 240.74 & $p<0.05$ \\
\hline
\end{tabular}

additionally tested $1 \%$ emulsified silicone oil for $24 \mathrm{~h}$, which also did not display any toxicity (data not shown).

\section{Toxicity of silicone oil-activated microglia cells on neuronal cell line PC12}

We tested the toxicity of silicone oil-treated microglia $(10 \%$ emulsified silicone oil, $72 \mathrm{~h}$ ) on a differentiated neuronal cell line, PC12 - untreated PC12 cells which served as controls, PC12 cells treated with the supernatant of untreated microglia, and PC12 cells treated with $10 \%$ silicone oil. We found no toxicity for either treatment. Of note, treatment with microglia medium, irrespective of the presence of silicone oil, induced a significant increase of the MTS signal (Fig. 3b) in Friedman's ANOVA and subsequent Wilcoxon's test, indicating an activating, but not toxic, effect on neuronal cells (Table 3 ).

\section{Uptake of silicone oil into microglia}

Intracellular silicone oil droplets were clearly detectable in light microscopy (Fig. 4a). We found time-dependent uptake of silicone oil droplets in microglial cells. A total of 12,562 cells were assessed (control 48 h, 2600 cells; 5\% silicone oil 48 h, 1387 cells; $10 \%$ silicone oil 48 h, 1237 cells; control 72 h, 3310 cells; $5 \%$ silicone oil 72 h, 2242 cells; $10 \%$ silicone oil $72 \mathrm{~h}, 1786$ cells). Untreated controls displayed few inclusions which fitted our definition of intracellular inclusions (72 h,
$0.0825 \% ; 48$ h, 0.269\%). Friedman's ANOVA showed a significant difference between the tested groups (48 h, $p<0.01$; $72 \mathrm{~h}, p<0.05)$. In subsequent Wilcoxon's $t$ test, a slight but significant uptake was found after $48 \mathrm{~h}$ for $5 \%$ and $10 \%$, a stronger uptake was found after $72 \mathrm{~h}$ for $5 \%$ and $10 \%$ (all $p<$ 0.05; Table 4) (Fig. 4b).

\section{Cytokine release of microglia after treatment with silicone oil}

In order to investigate a possible pro-inflammatory activation of microglia, we investigated the secretion of IL-6, IL-1ß, and TNF- $\alpha$ in ELISA after treatment with $10 \%$ silicone oil. Poly $\mathrm{I}: \mathrm{C}$ was used as a positive control.

No secretion of IL- 6 could be seen after 24 or $48 \mathrm{~h}$ of treatment with silicone oil (data not shown), while Poly I:C induced significant release of IL-6 after $24 \mathrm{~h}$ (Fig. 5a). After $72 \mathrm{~h}$, however, both control and silicone oil-treated cells showed secretion of IL-6, displaying a significant increase of IL-6 secretion between 72-h treatment and control $(p<0.05)$.

In contrast, a constitutive secretion of IL- 8 could be detected that increased with time. Incubation with Poly I:C for $24 \mathrm{~h}$ induced a strong increase of IL-8 secretion $(p<0.01)$. No difference between control and $10 \%$ silicone oil was found after 24 or 48 h. However, after 72 h, silicone oil-treated cells showed a significant increase in IL-8 secretion compared with untreated control $(p<0.05)$.

Table 3 Viability of PC12 cells after 72-h treatment with microglia medium treated with $10 \%$ emulsified silicone oil for $48 \mathrm{~h}$

\begin{tabular}{|c|c|c|c|c|c|c|c|c|c|}
\hline \multirow[t]{2}{*}{ Treatment } & \multirow[t]{2}{*}{ Rank } & \multirow[t]{2}{*}{ Rank sum } & \multirow[t]{2}{*}{ Median } & \multirow[t]{2}{*}{ Mini } & \multirow[t]{2}{*}{ Maxi } & \multirow[t]{2}{*}{ Friedman } & \multicolumn{3}{|l|}{ Wilcoxon } \\
\hline & & & & & & & vs (2) & vs. (3) & vs. (4) \\
\hline Micro/no silicon (1) & 2.8 & 14 & 109.69 & 92.64 & 118.06 & $p<0.01$ & $p<0.05$ & $p<0.05$ & n.s. \\
\hline Micro/10\% silicon (2) & 4 & 20 & 123.07 & 114.04 & 138.46 & & & $p<0.05$ & $p<0.05$ \\
\hline No micro/no silicon (3) & 1.3 & 6.5 & 86.29 & 66.55 & 100 & & & & n.s. \\
\hline No micro/10\% silicon (4) & 1.9 & 9.5 & 84.945 & 70.57 & 103.67 & & & & \\
\hline
\end{tabular}

Mini, minimum; Maxi, maximum; micro, microglia 
a) Exemplary picture of silicon droplet inclusions

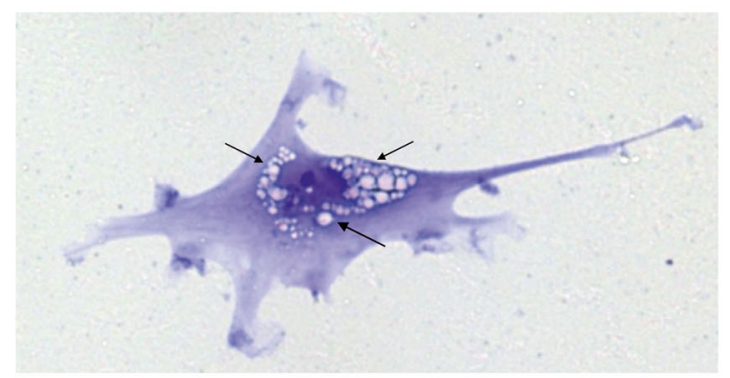

Fig. 4 Uptake of silicone oil droplets into microglia. Exemplary picture (a) shows clearly visible silicone oil droplets in microglia cells (arrows). Silicone oil droplets were taken up in microglia cells in a time- and concentration-dependent manner (b). Untreated microglia that were not

Only very little constitutive IL- $1 ß$ could be found after $24 \mathrm{~h}$ in microglia supernatant, but it was strongly induced by Poly I:C $(p<0.001)$. Constitutive secretion increased over time but did not change under treatment with $10 \%$ silicone after 24,48 , or $72 \mathrm{~h}$. A similar picture could be found for TNF- $\alpha$, with small amounts being constitutively found after $24 \mathrm{~h}$, a strong induction under Poly I:C, and some increase found over time with no significant changes under treatment with $10 \%$ silicone after 24,48 , or $72 \mathrm{~h}$.

\section{Discussion}

In this study, we investigated the effect of emulsified silicone oil on primary retinal microglia. Emulsification is one problem in the use of silicone oil tamponade. In long-term use, silicone oil droplets appear, most likely due to shear stress caused by eye movements. They can usually be found in the angle of the anterior chamber or even in the retina. In selected cases, usually with a history of several surgeries, they can be found in the optic nerve and even the brain $[2,3]$. We hypothesized for this study that silicone oil droplets interact with microglia which in turn may lead to microglia-mediated neurotoxicity [24], which might explain the phenomena of the unexplained loss in visual acuity under silicone oil. Emulsification of silicone oil was b) Uptake of silicone oil droplets in microglia cells

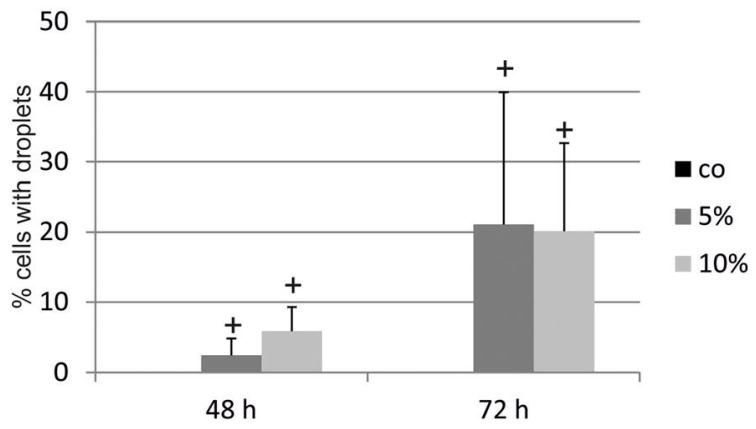

treated with silicone oil served as control and displayed few intracellular inclusions according to our definition (72 h, 0.0825\%; $48 \mathrm{~h}, 0.269 \%)$. Significance was evaluated with Friedman's ANOVA and Wilcoxon's test. Positive sign indicates $p<0.05$ compared with untreated control

achieved by sonication in supplemented medium, similar to previously described methods [25].

Inactivated microglia have a typical morphology with a small cell body and numerous extensions, while activated microglia change to an amoeboid state or may even turn into giant cells. Activated microglia may be phagocytic active and secrete cytokines. However, microglia can be activated to different degrees, depending on the stimulus and time scale of activation [19]. Our data clearly shows that silicone oil droplets exert an effect on microglia and that this effect is exercised over $72 \mathrm{~h}$. Due to the culturing condition, we did not exceed $72 \mathrm{~h}$ incubation time. We found that microglia took up silicone oil droplets which changed the metabolic status of the cells and induced secretion of IL-6 and IL-8. However, we found no significant changes in cell shape. As a comparison, we used stimulation with Poly I:C, a known activator of the Toll-like receptor (TLR)-3 and inductor of pro-inflammatory responses [15]. The response of our microglia in contact with silicone oil differed from pro-inflammatory-activated microglia treated with Poly I:C. While Poly I:C induced a general mobilization of cytokines, with an induction of IL-6, IL-8, IL$1 \beta$, and TNF- $\alpha$, the induction by silicone oil could only be detected for IL- 6 and IL-8. It is of interest that the induction of cytokines is not accompanied by a change in microglial morphology. This could be an indication that the silicon droplet uptake is not inducing a full-scale activation of the microglia

Table 4 Silicone oil droplets in microglia after treatment with $5 \%$ or $10 \%$ emulsified silicone oil

\begin{tabular}{lllllllr}
\hline Time $(\mathrm{h})$ & Treatment & Rank & Rank sum & Median & Minimum & Maximum & Friedman \\
\hline 48 & Control & 1.166667 & 7 & 0 & 0 & 3.23 & $p<0.01$ \\
& $5 \%$ & 2 & 12 & 4 & 0 & 15 & $p<0.05$ \\
& $10 \%$ & 2.833333 & 17 & 11 & 0 & 34 & $p<0.05$ \\
72 & 1 & 6 & 0 & 0 & 0.33 & $p<0.05$ \\
& Control & 15 & 15 & 15.48 & 1.84 & 45.63 & $p<0.05$ \\
& $5 \%$ & 2.5 & 15 & 19.71 & 5.22 & 44.99 & $p<0.05$ \\
\hline
\end{tabular}


IL-6

a) Poly I:C (24 h)

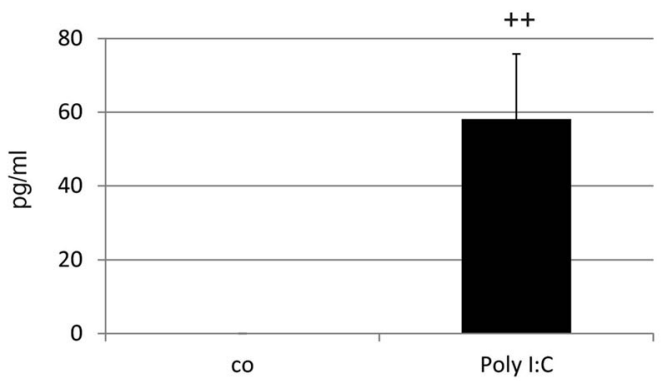

IL-8

c) Poly I:C (24 h)

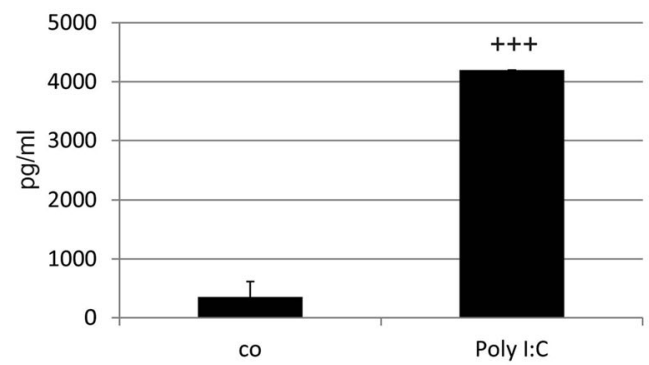

IL-1ß

e) Poly I:C (24 h)

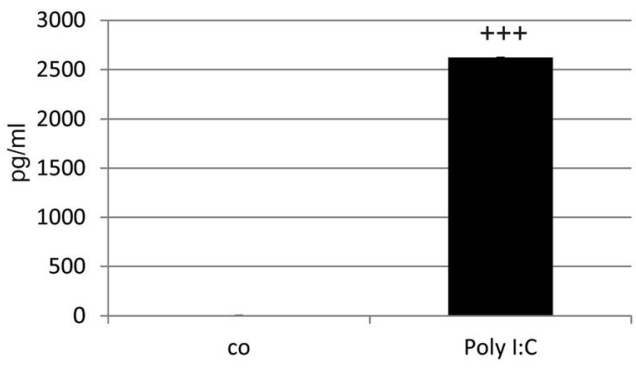

\section{TNFa}

g) Poly I:C (24 h)

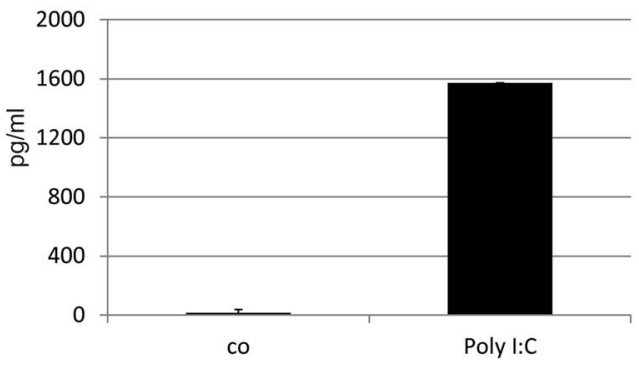

Fig. 5 Cytokine excretion by microglia incubated with $100 \mu \mathrm{g} / \mathrm{ml}$ Poly $\mathrm{I}: \mathrm{C}$ for $24 \mathrm{~h}(\mathbf{a}, \mathbf{c}, \mathbf{e}, \mathbf{g})$ or $10 \%$ silicone for $24 \mathrm{~h}(\mathbf{b}, \mathbf{d}, \mathbf{f}, \mathbf{h})$ and for 48 and $72 \mathrm{~h}$ (d, f, h). Poly I:C induced IL-6 (a), IL-8 (c), IL-1ß (e), and TNF- $\alpha$ (g) after 24 h. $10 \%$ silicone oil significantly induced IL-6 (b) and IL-8 (d)

but induce only a partial response. This would be in accordance with differentiated cytokine release we can see, with IL$1 \beta$ and TNF $\alpha$ not being induced by silicon droplets. Our data b) $10 \%$ silicone $(72 \mathrm{~h})$

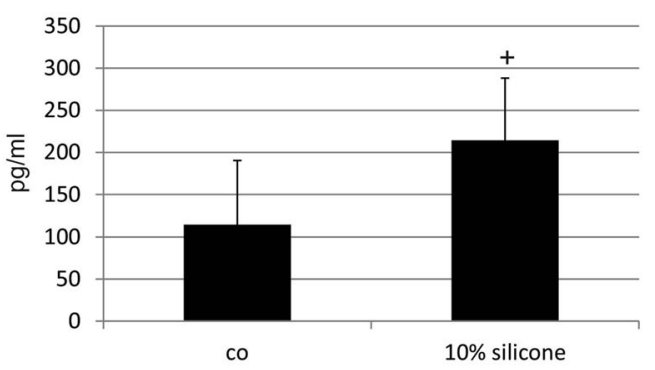

d) $10 \%$ silicone

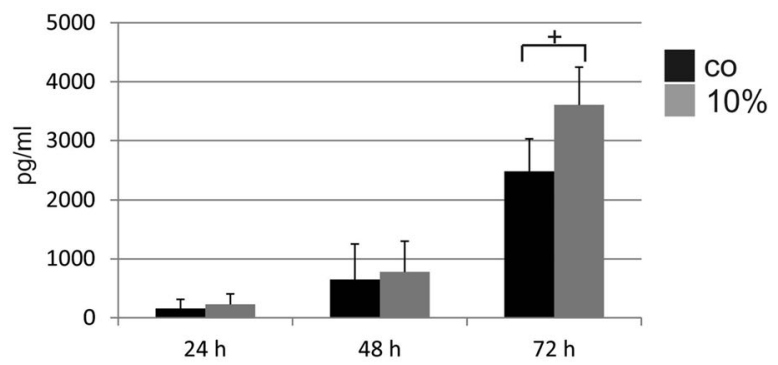

f) $10 \%$ silicone

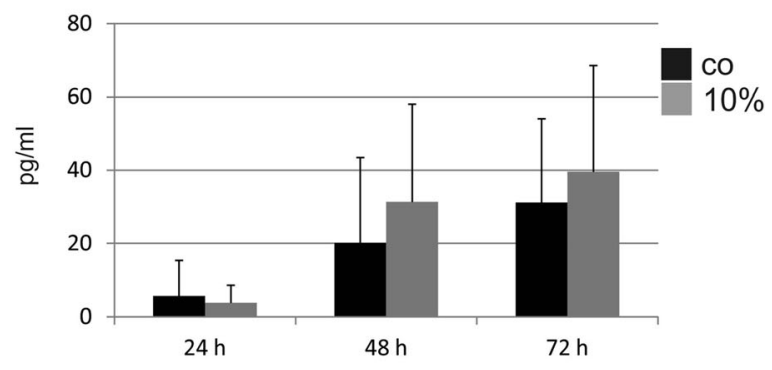

h) $10 \%$ silicone

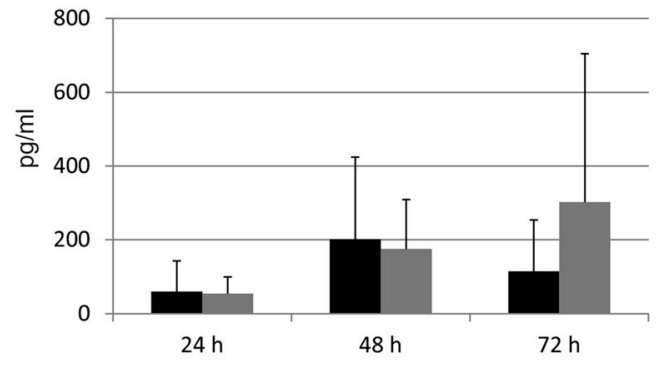

after $72 \mathrm{~h}$, but not IL-1ß or TNF- $\alpha$. Significance was evaluated with Student's $t$ test. Positive sign indicates $p<0.05$; Triple positive sign indicates $p<0.001$

suggests that silicone oil induces a differentiated effect. IL-1ß and TNF- $\alpha$ have been described to be neurotoxic [26], and their release by activated microglia can exert a direct toxic 
effect on neurons [27]. IL-6, on the contrary, can be neuroprotective, depending on receptor and signaling mechanisms [28, 29]. IL-8 primarily induces chemotaxis [30], but neurotoxic effects have been described for IL-8 as well [31]. Recently, elevated IL-8 levels have been correlated with acute vision loss and persistent dysfunction after acute optic neuritis [32]. Whether this mechanism is also relevant in the cases with unexplained loss in visual acuity after the use of silicone oil tamponade is speculative. A further evaluation of the levels of IL-8, e.g., in aqueous humor of patients with silicone oil tamponade in correlation with vision loss, would therefore be of high interest.

We further evaluated whether silicone oil-treated microglia exerted toxic effects on neuronal cells. Supernatants of silicone oil-treated microglia clearly did not induce cell death in differentiated PC12 cells, elevating the signal in the WST assay. As WST measures metabolic activity, our data clearly indicate that microglia exert an effect on neuronal cells, which, however, does not result in neuronal cell death, at least not in the measured time frame. The increase in the viability signal found both for microglia and PC12 cells could also indicate an activation of the cells in order to enable them to be more stress resistant, therefore, protective effects of the microglia on the neuronal cells could also be possible. Further research is needed to address this aspect.

Patients are treated with silicone oil for several months. We could show in our study that microglia react to silicone oil when it is in an emulsified state given an appropriate exposition time. Our data did not show any toxicity of microglia on neuronal cells after a 3-day exposure. Translated to the in vivo situation, this could imply that no acute toxicity is mediated by microglia in silicone oil-treated patients in the early period. This corresponds with the clinical data that usually do not see acute toxicity. It is difficult to transfer the time frame of cell cultures to the clinical time frame. Visual acuity loss usually occurs in the intermediate phase (6 weeks to 6 months) of the silicone oil tamponade. Our data would suggest that a longterm interaction of microglia with silicone oil might induce long-term pro-inflammatory changes and a long-term secretion of pro-inflammatory cytokines. As described in the literature, neurotoxicity of microglia usually develops when a proinflammatory stimulus cannot be eliminated [33]. An additional aspect that has to be considered is a priming of microglia. When primed, microglia will react much faster and more intense to an additional stimulus [19]. Translated to the in vivo situation, this could imply that patients treated with silicone oil for an extended time period may experience an otherwise milder noxious stimulus in a more neurotoxic manner.

Our data shows that retinal microglia react to emulsified silicone oil and our study clearly warrants further research on the long-term effect of silicone oil tamponades on retinal microglia and its consecutive effect on retinal neurons.
Funding Information Open Access funding provided by Projekt DEAL.

\section{Compliance with ethical standards}

Conflict of interest The authors declare that they have no conflict of interest.

Research involving human participants and/or animals This article does not contain any studies with human participants or animals performed by any of the authors.

Open Access This article is licensed under a Creative Commons Attribution 4.0 International License, which permits use, sharing, adaptation, distribution and reproduction in any medium or format, as long as you give appropriate credit to the original author(s) and the source, provide a link to the Creative Commons licence, and indicate if changes were made. The images or other third party material in this article are included in the article's Creative Commons licence, unless indicated otherwise in a credit line to the material. If material is not included in the article's Creative Commons licence and your intended use is not permitted by statutory regulation or exceeds the permitted use, you will need to obtain permission directly from the copyright holder. To view a copy of this licence, visit http://creativecommons.org/licenses/by/4.0/.

\section{References}

1. Lucke KH, Foerster MH, Laqua H (1987) Long-term results of vitrectomy and silicone oil in 500 cases of complicated retinal detachments. Am J Ophthalmol 104:624-633

2. Morescalchi F, Costagliola C, Duse S et al (2014) Heavy silicone oil and intraocular inflammation. Biomed Res Int 2014:574825

3. Grzybowski A, Pieczynski J, Ascaso FJ (2014) Neuronal complications of intravitreal silicone oil: an updated review. Acta Ophthalmol 92:201-204

4. Christensen UC, la Cour M (2012) Visual loss after use of intraocular silicone oil associated with thinning of inner retinal layers. Acta Ophthalmol 733-737

5. Tode J, Purtskhvanidze K, Oppermann T et al (2016) Vision loss under silicone oil tamponade. Graefes Arch Clin Exp Ophthalmol 254:1465-1471

6. Mrejen S, Sato T, Fisher Y, Spaide RF (2014) Intraretinal and intraoptic nerve head silicone oil vacuoles using adaptive optics. Ophthalmic Surg Lasers Imaging Retina 45:71-73

7. Federman JL, Schubert HD (1988) Complications associated with the use of silicone oil in 150 eyes after retina-vitreous surgery. Ophthalmology 95:870-876

8. Budde M, Cursiefen C, Holbach LM, Naumann GO (2001) Silicone oil-associated optic nerve degeneration. Am J Ophthalmol 131:392-394

9. Eckardt C, Nicolai U, Czank M, Schmidt D (1993) Ocular tissue after intravitreous silicone oil injection. Histologic and electron microscopy studies. Ophthalmologe 90:250-257

10. Papp A, Tóth J, Kerényi T et al (2004) Silicone oil in the subarachnoidal space-a possible route to the brain? Pathol Res Pract 200: $247-252$

11. Wickham LJ, Asaria RH, Alexander R et al (2007) Immunopathology of intraocular silicone oil: retina and epiretinal membranes. Br J Ophthalmol 91:258-262

12. Silverman SM, Wong WT (2018) Microglia in the retina: roles in development, maturity, and disease. Annu Rev Vis Sci 4:45-77 
13. Graeber MB, Streit WJ (2010) Microglia: biology and pathology. Acta Neuropathol 119:89-105

14. Klettner A, Hamann T, Schlüter K et al (2014) Retinal pigment epithelium cells alter the pro-inflammatory response of retinal microglia to TLR-3 stimulation. Acta Ophthalmol 92:e621-e629

15. Town T, Jeng D, Alexopoulou L et al (2006) Microglia recognize double-stranded RNA via TLR3. J Immunol 176:3804-3812

16. Tischler AS (2002) Chromaffin cells as models of endocrine cells and neurons. Ann N Y Acad Sci 971:366-370

17. de Los Rios C, Cano-Abad MF, Villarroya M, Lopez MG (2018) Chromaffin cells as a model to evaluate mechanisms of cell death and neuroprotective compounds. Pflugers Arch 470:187-198

18. Rudkin BB, Lazarovici P, Levi BZ et al (1989) Cell cycle-specific action of nerve growth factor in PC12 cells: differentiation without proliferation. EMBO J 8:3319-3325

19. Lull ME, Block ML (2010) Microglial activation and chronic neurodegeneration. Neurotherapeutics 7:354-365

20. Hornik TC, Neniskyte U, Brown GC (2014) Inflammation induces multinucleation of Microglia via PKC inhibition of cytokinesis, generating highly phagocytic multinucleated giant cells. J Neurochem 128:650-661

21. Riss TL, Moravec RA, Niles AL, et al. (2016) Cell viability assays. In: Sittampalam GS, Coussens NP, Brimacombe K, et al. (ed) Assay guidance manual. Bethesda (MD): Eli Lilly \& Company and the National Center for Advancing Translational Sciences

22. Peskin AV, Winterbourn CC (2000) A microtiter plate assay for superoxide dismutase using a water-soluble tetrazolium salt (WST1). Clin Chim Acta 293:157-166

23. Cai X, Ye T, Liu C et al (2011) Luteolin induced G2 phase cell cycle arrest and apoptosis on non-small cell lung cancer cells. Toxicol in Vitro 25:1385-1391

24. Brown GC, Vilalta A (2015) How microglia kill neurons. Brain Res 1628:288-279
25. Caramoy A, Schröder S, Fauser S, Kirchhof B (2010) In vitro emulsification assessment of new silicone oils. Br J Ophthalmol 94:509512

26. Ye L, Huang Y, Zhao L et al (2013) IL-1 $\beta$ and TNF- $\alpha$ induce neurotoxicity through glutamate production: a potential role for neuronal glutaminase. J Neurochem 125:897-908

27. Ma XC, Gottschall PE, Chen LT et al (2002) Role and mechanisms of interleukin-1 in the modulation of neurotoxicity. Neuroimmunomodulation 10:199-207

28. Bissonnette CJ, Klegeris A, McGeer PL, McGeer PL (2004) Interleukin 1alpha and interleukin 6 protect human neuronal SHSY5Y cells from oxidative damage. Neurosci Lett 361:40-43

29. Campbell IL, Erta M, Lim SL, Frausto R, May U, Rose-John S, Scheller J, Hidalgo J (2014) Trans-signaling is a dominant mechanism for the pathogenic actions of interleukin- 6 in the brain. $J$ Neurosci 34:2503-2513. https://doi.org/10.1523/JNEUROSCI. 2830-13.2014

30. Cross AK, Woodroofe MN (1999) Chemokines induce migration and changes in actin polymerization in adult rat brain microglia and a human fetal microglial cell line in vitro. J Neurosci Res 55:17-23

31. Thirumangalakudi L, Yin L, Rao HV, Grammas P (2007) IL-8 induces expression of matrix metalloproteinases, cell cycle and pro-apoptotic proteins, and cell death in cultured neurons. J Alzheimers Dis 11:305-311

32. Rossi S, Motta C, Studer V et al (2014) Interleukin-8 is associated with acute and persistent dysfunction after optic neuritis. Mult Scler 20:1841-1850

33. Cherry JD, Olschowka JA, O'Banion MK (2014) Neuroinflammation and M2 microglia: the good, the bad, and the inflamed. J Neuroinflammation 11:98

Publisher's note Springer Nature remains neutral with regard to jurisdictional claims in published maps and institutional affiliations. 